\title{
Estudios sobre opinión pública y proceso de paz entre el Estado Colombiano y las Farc-ep
}

\section{Studies on public opinion and the peace process between Colombia State and the Farc-ep}

DOI: http://dx.doi.org/10.17981/cultedusoc.11.2.2020.02

Recibido: 11 de noviembre de 2019 Aceptado: 12 de marzo de 2020 Publicado: 09 de julio de 2020

\author{
José Wilmar Pino Montoya \\ Universidad Católica Luis Amigó. Bello (Antioquia) \\ jose.pinomo@amigo.edu.co \\ Melina Castaño Gómez \\ Universidad de Antioquia. Medellín (Colombia) \\ jelicas17@gmail.com
}

Para citar este artículo:

Pino y Castaño (2020). Estudios sobre opinión pública y proceso de paz entre el Estado Colombiano y las Farc-ep. Cultura, Educación y Sociedad, 11(2). 27-42. DOI: http://dx.doi.org/10.17981/cultedusoc.11.2.2020.02

\section{Resumen}

La investigación considera como referente la preocupación sobre los diálogos de paz, desarrollados en la Habana, Cuba, entre el Estado Colombiano y las FARCep; de allí que el artículo tiene como propósito analizar las percepciones y opiniones de los colombianos sobre la referida iniciativa. Para abordar la situación objeto de estudio se privilegia el método cualitativo, con un tipo de estudio documental, a través del análisis de contenido que conduce a recuperar, sistematizar y valorar 24 estudios sobre la opinión pública y diálogos de paz. Los resultados se centran en artículos que destacan la categoría opinión pública a través de los medios de comunicación y su relación con los diálogos de paz, así como también el análisis de investigaciones que se ocupan expresamente de los procesos de paz en Colombia. En las conclusiones se aprecia como la opinión pública sobre el proceso de paz es construida desde una posición subjetiva por los medios de comunicación como encargados de cubrir los acontecimientos asociados a este proceso.

Palabras clave: Proceso de paz; opinión pública; Farc-ep; Democracia deliberativa; medios de comunicación

\section{Abstract}

The investigation considers as a reference the concern about the peace dialogues, developed in Havana, Cuba, between the Colombian State and the FARC-ep; Hence, the purpose of the article is to analyze the perceptions and opinions of Colombians on the referred initiative. To approach the situation under study, the qualitative method is privileged, with a type of documentary study, through content analysis that leads to the recovery, systematization and evaluation of 24 studies on public opinion and peace dialogues. The results focus on articles that highlight the category of public opinion through the media and its relationship with the peace dialogues, as well as the analysis of research specifically dealing with the peace processes in Colombia. In the conclusions it is appreciated how public opinion on the peace process is constructed from a subjective position by the media as being in charge of covering the events associated with this process.

Keywords: Peace process; public opinion; Farc-ep; Deliberative democracy; mass media 


\section{INTRODUCCIÓN}

El Estado Colombiano ha vivido momentos de transición y redefiniciones, en el caso de los conflictos, caracterizado por el signo de la guerra y otras manifestaciones de violencia, que han dejado indeseables e inconvenientes secuelas: el empeoramiento de los conflictos sociales; límites al progreso social y los problemas de convivencia civilizada. La compleja trama que configura esta realidad se fundamenta en múltiples causas y factores de origen interno y externo. Factores de tipo: económicos, políticos, culturales, locales e individuales que se ven reflejados en las diversas manifestaciones del conflicto del interno del país.

En este contexto, y según Valencia (2008), el Estado Colombiano ha tratado de darle fin al conflicto, especialmente con los grupos guerrilleros, de diversa manera, como y según Villarraga (2015) la tregua y el cese al fuego en el gobierno Betancur con las Farc-ep, el EPL y el M-19; en el gobierno Barco se implementaron los llamados pactos de paz con el M19, el EPL, el PRT y el MAQL; Gaviria propuso los acuerdo con el EPL y con varias fracciones guerrilleras y milicianas y los diálogos en Caracas y Tlaxcala; en el caso del periodo de Samper no hubo avance, ya que se fracasó con los diálogos propuestos con las Farc-ep y suspende las conversaciones iniciadas con el ELN; en el periodo del presidente Pastrana, si bien se propusieron y se comenzaron conversación con las Farc-ep y el ELN, estos no lograron su fin debido a su ruptura.

El presidente Uribe opto por una opción militar y su proceso se centró en los acuerdos con las AUC y la exploración de un acuerdo con el ELN; y por ultimo está, el caso que convoca este articulo y son los acuerdos implementados por el presidente Santos con las Farc-ep, con el cual se utilizó los procesos o diálogos de paz. Estrategia de paz con la cual se obtuvieron, en el pasado, resultados positivos con varios de los grupos guerrilleros, entre estos: el M-19, PRT, EPL entre otros.

Considerando, este contexto es que en el año 2012 el gobierno nacional propuso entablar un nuevo diálogo de paz con las Farc-ep, en la ciudad de la Habana (Cuba), en el que se proponían discutir 5 puntos a saber: 1) Política de desarrollo agrario integral; 2) Participación política; 3) Fin del conflicto; 4) Solución al problema de las drogas ilícitas; 5) Víctimas y verdad. Según la revista Semana (2012), los dos primeros son los reclamos históricos de las Farc-ep; los dos siguientes puntos se pueden entender como los reclamos de la sociedad; y el punto sobre víctimas y fin del conflicto tienen relación con el cómo se pone en práctica el acuerdo final.

Consecuentemente, el acuerdo de paz entre el gobierno y las Farc-ep tiene como fin "construir una paz estable y duradera, con la participación de todos los colombianos y colombianas. Con el propósito de poner fin de una vez y para siempre a los ciclos históricos de violencia y sentar las bases de la paz" (Acuerdo de paz, 2016, p. 6). Y es de vital importancia para el devenir y futuro del país, en lo que respeta a los acontecimientos que suscitaron el surgimiento de los grupos armados; la importancia de la desmovilización de las Farc-ep para la economía y los conglomerados económicos; la reorganización de las zonas de conflicto y la esperanza de la sociedad civil y de la población en general de no sufrir nuevamente las consecuencias y las huellas que dejó la guerra, es que resulta relevante realizar un estudio de las opiniones suscitadas ante los diálogos de paz relacionadas con variables económicas, políticas, ideológicas o sociales de quienes se pronuncian al respecto. 


\section{REFERENTES TEÓRICOS}

Los referentes teóricos que fundamentan el proceso de análisis está compuesto por cinco grades categorías: paz, proceso de paz, acuerdo de paz, opinión pública y democracia deliberativa, como conceptos relacionados para dar coherencia al escrito.

Sobre el concepto de paz y retomado en primera instancia a Jiménez (2009), se aprecia:

En términos históricos, se han dado tres maneras de estudiar el concepto de paz. Paz como paz negativa (ausencia de violencia directa); paz positiva (ausencia de violencia estructural o indirecta (...) Y, en últimas fechas, se menciona la paz neutra (ausencia de violencia cultural y/o simbólica)". (p. 146)

No obstante, Galtung (2003) amplía y redefine el concepto y reconocen que la paz tiene dos lados, uno negativo y otro positivo. Galtung, llama paz positiva al «despliegue de la vida» y paz negativa a la superación de las tres formas de violencia, directa, estructural y cultural. Es así como:

La creación de la paz tiene que ver, obviamente, con la reducción de la violencia (cura) y con su evitación (prevención). Y violencia significa dañar y/o herir. Por lo tanto, asumimos la existencia de algo que puede experimentar el ser dañado y ser herido, y seguimos la tradición budista al identificar ese algo como la vida" (Galtung, 2003, p. 20).

Considerando los alcances y aportes de los anteriores conceptos, es pertinente y para este trabajo contar con el concepto de "Paz Transformadora" que acuña Ramos (2015) como "el proceso mediante el cual los seres humanos cristalizamos, transductivamente, modelos convivenciales de atención sinérgica de las necesidades para el conjunto de la población. Esto es, modelos convivenciales de Buen Vivir" (p. 48).

\section{Proceso de paz y acuerdo de paz}

Los procesos de paz según Darby (2001), citado por Ugarriza, Cotrina y Sequera (2013) implican "un concepto extenso que se refiere al esfuerzo de los actores de un conflicto por hallar una solución, y abarca elementos como las exploraciones iniciales entre las partes, las negociaciones, los acuerdos y su implementación”. (p. 154). Estos procesos de paz precisan que las partes negocien de buena fe, además se deben incluir todos los actores clave. Por otra parte, estos procesos de paz pueden terminar en fracaso si no se tocan los puntos centrales de la agenda; si hay un uso indebido de la fuerza por alguna o ambas partes y si no hay un compromiso a largo plazo. Por otra parte, y como nuevamente los reiteran Ugarriza et al. (2013) no existe procesos de paz sin una negociación de una agenda importante entre los actores del conflicto. Partiendo de esa definición, los autores hacen una diferencia entre los conceptos de exploración, negociación, proceso y acuerdo de paz.

El término exploración, o acercamiento, aplica a un número amplio de episodios entre actores de un conflicto que no necesariamente se refiere a un proceso de paz. [...] Las exploraciones pueden evolucionar hacia negociaciones que, sin embargo, tampoco implican un proceso de paz [...] sólo la aparición de una agenda sustantiva y dirigida a poner fin al conflicto convierte una negociación en un proceso de paz (Ugarriza et al., 2013, p. 154). 
En este aparte vale la pena anotar que solo dentro de un denominado proceso de paz, puede hablarse de acuerdos de paz y no desde cualquier tipo de negociación. Para Darby (2001), citado por Ugarriza et al. (2013), en todo proceso de paz deben intervenir todos los actores clave, incluidos la sociedad civil, incluyendo lo que estos piensan y opinan: sobre cómo se debe implementar; como llevar un acuerdo de paz; y si están de acuerdo de utilizar esta alternativa para terminar y solucionar el conflicto.

\section{Opinión publica. Conceptualizaciones básicas}

Sobre el termino opinión pública, dice Aparicio (2004):

La opinión pública se presenta como el conjunto de ideas que pueden expresarse en la esfera de lo público a través de los Medios de Comunicación Social (MCS), sin miedo al aislamiento o a la coacción, dado que para ejercerla es necesario detentar algún tipo de poder (económico, político, religioso, civil u otro); de allí, que se considere un fenómeno social al amparo de la comunicación política (p. 322).

Si bien el anterior argumento, liga a la opinión pública con algún tipo de ostentación de poder, en las sociedades que propugnan por que los individuos sean iguales ante la ley, este concepto está caracterizado por garantizar la participación igualitaria en las decisiones del gobierno.

Las tesis democráticas y socialistas, al exigir que la igualdad ante la ley signifique igual participación en la producción de la norma y del gobierno y al entender el fin de la participación, de la norma y del gobierno como un proyecto de organización de toda la sociedad en su conjunto sobre bases igualitarias sustanciales (económicas y sociales), desencadenaron necesariamente la pluralidad de ingenierías constitucionales, planes políticos, programas de acción administrativa y, en consecuencia, pluralidad de la opinión pública (Aguilar, 2017, p. 136).

En este orden de argumentos se podría, invocar a Sartori (1998), cuando precisa que

Debemos añadir que una opinión se denomina pública no sólo porque es del público, sino también porque implica la res publica, la cosa pública, es decir, argumentos de naturaleza pública: los intereses generales, el bien común, los problemas colectivos (p. 69).

En la opinión pública y en la garantía que ofrecen los Estados modernos para ejercerla, se sustenta la llamada democracia deliberativa. En este tipo de democracia, tal y como lo afirma Sampedro (2000) citado por Pino (2016) “(...) los ideales de participación y decisión colectiva se saben siempre inacabados (...) la democracia deliberativa se alcanza tomándose en serio los derechos de participación ya existentes" (pp. 25-26).

Cabe entonces concluir que la democracia deliberativa está fundamentada en la opinión pública y una participación activa de la sociedad civil.

\section{MetodoloGía}

Para efectos de esta investigación se privilegió la investigación cualitativa. Desde lo cualitativo "no sólo es una técnica de recolección y validación de información, sino que constituye una de sus estrategias, la cual cuenta con particularidades propias en el diseño (...), la obtención de información, el análisis y la interpretación” (Galeano, 2004, p. 114); por ello 
se convierte en el tipo de investigación adecuada para recuperar, reconocer y valorar los estudios que se han realizado en la temática. Esta investigación, además, parte de indagar algunos "referentes teóricos" relacionados con el tema, es decir, una fuente de información bibliográfica que permitirá operatividad y prestar especial atención a las categorías y los esquemas de análisis. De igual modo, permitió contrastar los hallazgos y construir las conclusiones derivadas del análisis de las indagaciones obtenidas al utilizar y complementar las técnicas del enfoque cuantitativo.

Este estudio se realizó en tres momentos, el primero de indagación y recolección de la información: en primer lugar, una codificación sustantiva o abierta pasando luego a un segundo nivel de codificación selectiva o axial. Se Identifican los conceptos que muestran alguna conexidad y se hace una revisión de la literatura para generar; luego se pasa a la fase de interpretación y análisis, allí se categorizaron los datos conforme a patrones y tendencias que se descubrían tras la lectura de los mismos, desde este punto de vista se utilizaron como herramientas fichas analíticas (descriptivas y de contenido) que se construyen como memos con los que se sintetiza las explicaciones teóricas de los textos y contextos. Además, se implementaron las matrices que sirven también de herramienta para diagramar y trazar en un mapa las interrelaciones clarificando y habilitando un creciente nivel de abstracción. Para algunos hallazgos fue de mucha utilidad el fichero como herramienta analítica e interpretativa.

\section{HALLAZGOS Y ANÁLISIS}

\section{Medios de comunicación y proceso de paz}

Frente a las investigaciones e indagaciones que se ha realizado sobre: Opinión pública y proceso de paz se puede nombrar en primer lugar el de Bello (2014) de la Universidad Austral de Buenos Aires Argentina, quien en su tesis doctoral titulada: "Periodismo y violencia política en Colombia. Una mirada crítica desde el Periodismo de Paz", centra la atención en "realizar [un] estudio con el propósito descubrir, analizar y explicitar desde el discurso periodístico el cómo de la intención de los partidos conservador y liberal de establecer una hegemonía del poder" (p. 14). Para hacer este análisis se tomó como base la coincidencia de la promulgación de la conquista de la paz de los presidentes: Guillermo León Valencia en 1964, con la Operación Marquetalia, y Álvaro Uribe Vélez en 2008 con la muerte del Raúl Reyes y cuyas revelaciones que tuvieron trascendencia en las notas editoriales de los periódicos El Tiempo y El Colombiano, que a la postre, dichos acontecimientos no lo lograron la paz, meta que, según el autor, aún está por alcanzarse. Sobre medios de comunicación y proceso de paz, en 2015, Bedoya y Londoño publicaron el estudio: "Proceso de paz colombiano en prensa y revistas: un análisis desde la aproximación histórica discursiva y la argumentación pragmadialéctica”. Sobre el propósito de este material, dicen los autores que:

(...) el presente artículo acude a la aproximación histórica discursiva y a la argumentación pragmadialéctica como medios para analizar en algunos articulistas de periódicos y una revista de amplia circulación en Medellín (Antioquia) elementos de manipulación y las cargas ideológicas que se presentan en un discurso (...) (p. 82). 
"Opinión pública y proceso de paz en Colombia en 2014" es un artículo publicado también, por el antropólogo Charry (2004). En este trabajo el autor analiza "los medios de comunicación como un actor esencial del proceso de construcción de la opinión pública; por esa razón, su punto de vista es una clave para entender los diálogos de paz, así como el desarrollo del conflicto armado en Colombia (p. 510).

Otro de los artículos encontrados, sobre este tema, fue el de Grajales y Martínez (2020) de la Corporación Universitaria Minuto de Dios (Uniminuto), denominado: "Tendencias, trayectorias y relaciones de poder en el discurso de construcción de paz en el periódico El Espectador (Colombia)”. Este artículo:

(...) analiza las tendencias, trayectorias y relaciones de poder producidas en el discurso de construcción de paz de El Espectador en 3 hitos del proceso de paz adelantado en Colombia entre 2012 y 2016: inicio de diálogos, su suspensión y realización del plebiscito (p. 112).

Este trabajo permite evidenciar como este medio, aunque avala los diálogos, se muestra de acuerdo con el uso de la violencia por parte del Estado.

Por otra parte, se encuentra el trabajo de Gómez (2017) de la Universidad del Rosario: "Periodismo de Paz: formar el camino hacia el final del conflicto". Esta autora "analiza el cubrimiento periodístico de dos hitos mediáticos en el proceso de paz del gobierno de Juan Manuel Santos con la guerrilla de las FARC-EP, la firma del acuerdo de justicia transicional y la firma del cese al fuego (...).

El trabajo invita reflexionar sobre el papel de los medios de comunicación en la búsqueda de soluciones pacíficas a los conflictos y proponer el periodismo como una manera de reflexionar la paz.

Para "analizar el discurso audiovisual de Noticias RCN de las 7:00 p.m., durante las campañas del Plebiscito desde el 24 de agosto al 2 de octubre de 2016 para así identificar cómo uno de los medios de comunicación con más teleaudiencia en Colombia, participó en la construcción de cultura política al rededor del Acuerdo de paz”. López y Ramírez (2017), para optar al título de Licenciatura en Artes Visuales, hacen el trabajo: "Interferencias en dialogo: Representación de la cultura política en mediaciones televisivas, en el marco del Acuerdo de Paz en Colombia”. Con este trabajo se concluyó que, en medio de la coyuntura política motivada por los acuerdos de paz, los medios de comunicación participaron de manera activa en la formación de opinión pública, reforzando así la configuración de la cultura política de los colombianos sobre el proceso de paz.

Del mismo modo Gómez (2009) de la Universidad de la Tadeo Lozano, realiza su trabajo sobre la manera como la construcción de paz es manejada por los diversos medios de comunicación, con el artículo: "Medios de comunicación en la construcción de paz". Allí, "Se [hace] especial énfasis en el papel que juega la comunicación, entendiendo esta como ente formador de opinión pública y concibiendo el gran impacto que tienen los medios en la construcción de paz (Gómez, 2009, p. 19). Un quinto artículo es el de Cárdenas (2015) de la Universidad de la Sabana, el cual se tituló: "Los medios de comunicación y la construcción de la opinión publica del proceso de paz de La Habana: algunas reflexiones sobre el papel de los medios en la construcción mediática de la realidad en torno a la paz en Colombia”. Este artículo, trata sobre como "la opinión pública es una variable fundamental a estudiar en los procesos de negociación de paz como el que se adelanta en Colombia” (p. 3). 
La reflexión pretende hacer un análisis en contexto de la evolución de las opiniones de los bogotanos frente al proceso de paz a partir de los resultados de una serie de estudios de opinión realizados entre el año 2013 y 2014 por el Observatorio de Medios de la Universidad de la Sabana (Cárdenas, 2015, p. 3).

Dentro de este mismo bloque temático se puede mencionar el trabajo de Ayala (2015) quien hace un estudio sobre las posturas editoriales, asumidas por la prensa colombiana sobre el inicio y devenir del proceso de paz, en su ensayo: "El proceso de paz de la habana y las posturas editoriales de la prensa colombiana".”. Lo que se encontró es que hay diferencias en las posturas asumidas por los tres medios de comunicación estudiados: El País, El Tiempo y El Espectador, evidenciando que históricamente, estos medios tienen una clara postura ideológica, lo que les define su postura frente a los diálogos de paz: El Tiempo y El Espectador apoyando el proceso de paz y El País asume unas posturas ambivalentes, un poco más ambivalente.

En esta misma perspectiva, se menciona el trabajo de Castellano, Virviescas, Castro, Albarino, Pinzón y Gutiérrez (2017): "Resolución de conflictos para el fomento de la cultura de paz: importancia de los medios de comunicación alternos en Colombia" quienes hacen una investigación para "analizar la resolución de conflictos para el fomento de una cultura de paz, mediante la intervención de los medios de comunicación alternativos” (p. 56). Este es un acercamiento a los aportes a la resolución de conflictos desde los medios de comunicación que no son manejados por estructuras de poder.

En este aparte también cabe mencionar la investigación realizada por Pedroza (2011), "denominada opinión pública en Colombia: Análisis de los medios de comunicación: Semana y Cambio en el cubrimiento periodístico del tema de justicia y paz durante el año 20052006”. En este texto la autora hace un recorrido teórico sobre los conceptos de opinión pública abordados desde una perspectiva histórica hasta llegar al análisis de la teoría en la relación con los medios de comunicación y como estos influyen en la opinión pública, tomado como tema específico la ley de justicia y paz. Conviene aquí sobresaltar el artículo: "El tratamiento del postconflicto colombiano por medio de infografías y visualizaciones de datos". Aquí se pretende mostrar cómo y a través de diferentes medios y para validar lo discutido en la Habana "el gobierno y algunas entidades no gubernamentales implementaron estrategias para dar a conocer los acuerdos de todas las maneras posibles" (p. 700).

De allí que este trabajo, se propuso analizar las formas narrativas y los múltiples discursos empleados, así como el aprovechamiento de herramientas digitales empleadas para informar a la sociedad el alcance de lo que se estaba pactando.

\section{Opinión publica sobre procesos de paz}

Después de haber dedicado la primera parte de estos hallazgos al tema de medios de comunicación y proceso de paz, ahora se centrará la atención en lo relacionado las investigaciones realizadas sobre opinión pública sobre los procesos de paz.

Desde esta perspectiva de análisis, Marín y Quintero (2018) publican el artículo. "Confianza en el proceso de paz en Colombia en Twitter", como “(...) resultados de una investigación sobre las expresiones de confianza en la red social Twitter relativas al proceso de paz entre el gobierno de Colombia y las farc-ep" (p. 115). En esta investigación se analizaron dos categorías: acuerdos de paz y paz. En los resultados se muestra que para los participantes 
los acuerdos de paz resultan insuficientes, ya que la paz real depende de las actitudes de los ciudadanos.

Sobre opinión pública, también hace referencia la encuesta que realizó la Fundación Social (Montoya, 2005), la cual se tituló: "percepciones y opiniones de los colombianos sobre justicia, verdad, reparación y reconciliación”. La investigación se centró en conocer la percepción que tiene la población colombiana, sobre los temas de justicia, verdad, reparación y reconciliación. A esta población, quien era la muestra representativa de los hogares residente en las cabeceras municipales del país, se le aplicó una encuesta, Esta muestra permitió el análisis por regiones, estratos, nivel educativo, sexo y edad. Muy similar al anterior tema de indagación, se encuentra el estudio que realizó Cárdenas (2013) en Bogotá, desde un método cuantitativo, titulado: “opinión pública y proceso de paz: actitudes e imaginarios de los bogotanos frente al proceso de paz de La Habana entre el gobierno colombiano y la guerrilla de las FARC", entre sus hallazgos se encuentra.

Que dentro del imaginario colectivo de la paz prime el valor de la tranquilidad, entendiendo la paz como la ausencia de disrupciones al orden y la calma pública (Ver diagrama 5). Sin embargo, el imaginario se alimenta también de valores conceptuales más orientados hacia la comprensión de la paz positiva como la libertad y la igualdad, y de valores conceptuales dirigidos al restablecimiento de la convivencia, como el respeto, la tolerancia y la armonía (p. 57).

Se puede nombrar en este punto, el artículo de Villa y Arroyave (2018), denominado: "Creencias sociales y orientaciones emocionales colectivas sobre la paz negociada en ciudadanos de Medellín. . En este artículo presenta los resultados de una investigación que "se ha desarrollado con ciudadanos de la ciudad de Medellín, en el contexto y el marco del proceso de negociación política del conflicto armado entre el gobierno de Juan Manuel Santos y las Fuerzas Armadas Revolucionarias de Colombia (FARCEP) (p. 450). Los autores analizan desde las experiencias, orientaciones emocionales colectivas y creencias sociales los aportes de los participantes en la construcción de paz, pues este es uno de los acontecimientos más relevantes de los últimos años en Colombia.

Igualmente es significativo resaltar el artículo de Ramírez, Ceballos y Molina (2019), de la Universidad del Valle, titulado: "Actitudes de la población vallecaucana frente a la convivencia y reconciliación en el proceso de paz entre el Gobierno Colombiano y las Fuerzas Armadas Revolucionarias de Colombia (FARCEP”. Los profesores hacen una investigación con el "objetivo identificar la relación entre la actitud de la población vallecaucana frente a la responsabilidad en el proceso de reconciliación y el eventual proceso de convivencia que tienen los actores del conflicto armado-político de Colombia" (p. 3). entre los resultados más relevantes se aprecia que:

Respecto a la actitud de la población vallecaucana frente a la responsabilidad que ésta tiene en el proceso de reconciliación y que le atribuye a los actores del conflicto armado se observó que dos grupos de la población mantienen una estabilidad en su actitud, el primero al mostrar favorabilidad en que todos los actores del conflicto armado son responsables del proceso de reconciliación y, el segundo al mostrar desfavorabilidad frente a la responsabilidad que la población civil y las víctimas tienen en dicho proceso (Ramírez et al., 2019, p. 14).

Otro de los autores que se ha dedicado al tema de la opinión pública, ahora desde las acciones participativas es Basset (2018), con su escrito "Claves del rechazo del plebiscito para la paz en Colombia”. 
El artículo analiza la victoria del NO en el plebiscito sobre los Acuerdos de Paz de octubre de 2016 en Colombia, mediante la cartografía analítica. Muestra la existencia de un clivaje territorial estable acerca del conflicto armado que se expresó en las urnas tanto en la segunda vuelta presidencial de 2014 como en el plebiscito, y se enfoca en explicar los cambios decisivos entre los dos eventos electorales que fueron decisivos en la victoria del NO (p. 241).

En este artículo la principal conclusión destaca "que la victoria del NO se debe al voto de los sectores populares urbanos, periurbanos y de las ciudades intermediarias que no se sintieron interpretados por el discurso de la paz y temieron ser olvidados en el contexto del posconflicto" (Basset, 2018, p. 241). Se observa como a través de la manifestación en las urnas los ciudadanos pudieron opinar en pro o en contra de los diálogos de paz de hecho; esto se corresponde con lo expuesto por Cifuentes (2006), quien en su artículo presenta el papel que puede cumplir la ciudadanía dentro del marco de una democracia deliberativa.

Allí, la opinión pública se presenta en el modelo democrático deliberativo, modelo de gobierno que centra su atención en el interés de legitimarse en el dialogo y en la función que cumple el poder de la palabra y la habilidad comunicativa dentro de un sistema político administrativo. Sobre, este mismo tema, de la opinión pública y su importancia para la democracia, Sopena (2008), proporciona una investigación sobre las líneas de investigación en opinión pública en Europa, considerando que esta es un elemento capital para el sostenimiento de las democracias del siglo XXI. Desde otro punto de énfasis Carranza (2007), en su artículo: "Los acuerdos de paz, 15 años después. Una mirada desde la opinión pública" da cuenta de cómo la opinión pública califica los avances o retrocesos en el cumplimiento de los acuerdos de paz, en el Salvador después de 15 años de su firma.

Entre otros autores que se ha interesado por investigar la relación entre opinión pública y democracia, se menciona a Morales, Rodríguez, Reyes y O'Quinn (2011). Ellos escriben un artículo titulado: "opinión pública y democracia, algunas aportaciones para su estudio". El propósito de este artículo es reflexionar sobre la importancia que tiene la opinión pública desde el punto de vista teórico conceptual con el fin de que se puedan apreciar con claridad los diversos elementos que la configuran y con ello saber la evolución en la concepción moderna.

Entre las investigaciones que se preocupan por indagar por los procesos de paz, se pueden contar la de Cabrales (2012); este autor presenta el artículo: "La justicia y paz y el marco legal para la paz. Un paso más hacia la impunidad”. Sus hallazgos consisten en hacer primera instancia, una aproximación a las causas de la perpetuación del conflicto armado en Colombia; posteriormente analiza la ley de justicia y paz luego hace una lectura crítica de la política de perdón y olvido como una manera de promover la impunidad y de cómo esta estrategia ha llamado la atención de la opinión. En este sentido se encuentran, además los estudios hechos por Calderón (2016) en su trabajo denominado "Etapas del conflicto armado en Colombia: hacia el posconflicto", donde:

Analiza las etapas en las que se ha desarrollado el conflicto armado colombiano desde una perspectiva teórica la cual establece que un conflicto tiene su propio ciclo de vida, que alcanza un punto máximo e incluso violento, luego disminuye, desaparece y en algunos casos reaparece (Calderón, 2016, p. 227). 
En este orden de ideas el autor llega a la conclusión que el conflicto en Colombia, ha pasado por tres etapas: una inicial, la intermedia y la final. Cada etapa tiene sus propios retos, pero la etapa final o la de posconflicto, es la más larga, costosa y la de mayores retos.

Por su parte, "Justicia, verdad y reparación en el proceso de paz en Colombia", es un escrito de Gómez (2016), donde se concluye:

Uno de los principales retos del proceso de paz entre el Gobierno colombiano y las farc-ep es la conciliación entre el logro de la paz y la garantía de los derechos de las víctimas a la justicia, a la verdad y a la reparación (p. 35).

En este artículo el autor hace amplias referencias al marco jurídico para la paz, el cual es definido como un conjunto de mecanismos de justicia transicional que tiene como pretensión facilitar y garantizar las negociaciones, evitar la impunidad y otorgar garantías a las víctimas.

En el marco de este mismo tema, se encuentra el artículo: "Caracterización de los crímenes de estado y el proceso de paz en Colombia", producto de una investigación, hecha por Libreros (2018). Aquí el autor "destacará que la búsqueda de la paz ha sido un anhelo universal en todos los tiempos. Lo evidencian los numerosos procesos de justicia transicional acaecidos en diferentes países del mundo, y Colombia no es la excepción (p. 119).

Por otra parte, el escritor precisa que Colombia, después de muchos intentos para alcanzar la paz, logra hacerlo con la firma del acuerdo en la Habana y se resalta que, en el marco del conflicto, el Estado cometió varios excesos, avalados por el ejecutivo. Palacio (2017) con su trabajo "Perspectivas y comparaciones en los diálogos de paz en Colombia", le hace otro aporte a este tema de estudio centrado en el proceso de paz. El ensayo es un ejercicio analítico que propone entender el actual proceso de paz, con el que se espera acabar con un conflicto percibido como el más largo del mundo occidental. Busca delimitarlo, definir sus características, matices, su génesis y sus actores. Este punto lo complementa Ramos (2016), cuando en su artículo "El proceso de construcción de paz colombiano más allá de la negociación: una propuesta desde la Paz Transformadora y Participativa”.

Analiza el proceso de paz que se adelanta en Colombia entre la insurgencia de las FARCEP y el Gobierno de Colombia, observado desde los enfoques teóricos de los Estudios de la Paz, y desde la perspectiva del movimiento social, argumentando la necesidad de asumir el enfoque epistemológico de la Paz Transformadora y Participativa como vía para propiciar procesos integrales de construcción de paz más allá de la negociación (p. 513).

En este artículo, la principal conclusión se orienta a "impulsar una estrategia coordinada de construcción de paz como un esfuerzo colectivo por y para los pueblos de Colombia es posible y necesario" (p. 528).

Por último y para terminar esta sesión del trabajo, es importante resaltar el estudio que hace Villarraga (2015), titulado "Los procesos de paz en Colombia, 1982-2014”. Este trabajo recoge de manera precisa la memoria histórica de los procesos de paz en Colombia con el fin de divulgar y de servir de ayuda pedagógica, publicando de manera concisa que:

El conjunto de las políticas gubernamentales de paz, los eventos de diálogo y negociación entre gobiernos nacionales y los movimientos guerrilleros, los acuerdos conseguidos y las principales dinámicas asociadas a los diversos procesos de paz que han tenido lugar en el período de tiempo señalado (Villarraga, 2015, p. 9). 
Su contenido retoma apartes relacionados con los ensayos construidos por el mismo autor y los complementa con presentaciones de las acciones realizadas por los movimientos sociales y ciudadanos por la paz en Colombia durante últimas tres décadas, además hace un aporte sobre el proceso de paz, entre el gobierno colombiano y las FARC-EP, que está en curso en La Habana, complementa su análisis haciendo alusión a la fase exploratoria con el ELN.

\section{DisCUSIÓN}

Uno de los mecanismos o espacios donde los ciudadanos pueden exponer críticamente su postura y su opinión, es decir expresar de manera crítica lo que piensan de sus gobiernos y sus actuaciones es a través de su opinión o manifestación pública; su participación en debates y espacios donde libremente digan lo que piensan. "En este sentido, su posible tarea dentro de las democracias poliárquicas es configurar acumulativamente la opinión de los ciudadanos para el momento de la elección de los decisores" (Aguilar, 2017, p. 147). Es aquí donde el término de opinión pública, toma relevancia desde sus dos acepciones: el primero está asociado con como lo afirma Gómez (2009) con:

Un tipo de conocimiento de los individuos sobre su entorno, conocimiento distinto del científico. La segunda cuestión se refiere a su carácter de "pública". Lo público puede remitir a una sociedad no cerrada, al acceso general, a la administración del Estado, a la representación, al reconocimiento, incluso a la publicidad, etc. (p. 27).

Con respeto a la relación entre opinión pública y los diálogos de paz, la información que obtiene la población en general es básicamente de los medios de comunicación, que como se aprecia en este estudio, la opinión sobre los diálogos de paz se concentró en su mayoría en medios periodísticos, quienes ideológicamente y a partir de sus intereses brindaban la información. "La construcción de opinión sobre los diálogos de paz es construida a partir de medios de comunicación de masas como la televisión, la prensa escrita y en especial el uso de los medios digitales como el internet" (Pino, 2016, p. 92).

Por esta razón el público en general y para formarse una percepción adecuada del proceso de paz debió haber tenido múltiples alternativas para lograr la información, no obstante, y como se encontró, se tuvo que conformar con las noticias publicadas por los medios tradiciones de comunicación como la prensa y los noticieros de televisión que son manejados por los organismos de poder. De este modo, estos medios influenciaron y se encargaron de informar a la ciudadanía sobre este tema de coyuntura lo que puede generar uno u otro tipo de opinión entre las personas. Como bien lo afirma Cárdenas (2013) cuando dice que:

Los efectos que puede tener los medios de comunicación sobre la formación de la opinión pública frente a un tema van desde la capacidad de visibilizar o invisibilizar el tema, es decir hacerlo parte de la agenda, construir marcos de interpretación "frames" frente a los temas de la agenda, y establecer las voces autorizadas y fuentes más confiables o "reputadas" para hablar de los temas de la agenda desde los marcos de interpretación construidos (p. 46).

El anterior argumento lo corrobora el artículo de D'Adamo, García y Freidenberg (2000), denominado efectos políticos de los medios de comunicación, centra más su atención en como a través de los medios de comunicación se manipula la opinión pública y como esta manipulación tiene efectos políticos en los electores, creándoles o formándoles una falsa percepción de los acontecimientos. Allí, y contrario a las investigaciones antes mencionadas, los autores analizan la función del establecimiento de la agenda en el campo de la comunicación pública. 
Un elemento muy importante en esta investigación es el papel de los medios de comunicación en la percepción que la opinión pública construye de las cuestiones públicas y políticas y como estas manipulan y acomodan la información. Así pues "La construcción de actitudes frente al proceso de paz está influenciada por el papel que los medios de comunicación y los actores del proceso puedan tener en la construcción mediática de la realidad política" (Cárdenas, 2013, p. 45).

Por otra parte, y como se aprecia en la última categoría (Opinión pública y diálogos de paz), también existe estudios que demuestran que existe otra opinión pública y es la expresada por las personas del común, que tienes una opinión del proceso de paz pero construida en el dialogo cotidiano y con la relación de las personas más cercanas:

La opinión pública, tiene dos vías principales para su formación: la sociedad y los medios de comunicación. En la primera, tiene un gran peso la familia, la escuela y los grupos sociales, en la segunda; se considera que los medios evocan los temas sobre los que una sociedad debe tener prioridad y sobre aquellos que debe reflexionar, de ahí la importancia en la formación de actitudes y por consiguiente de opinión (Glynn, Herbst, O'Keefe, Shapiro \& Lindeman, 1999, p. 6).

Este tipo de opinión pública es entonces, fundamentada en el dialogo, en la exposición frente a frente de los argumentos y de las razones y como junto al poder comunicativo tiene influencia en el sistema político administrativo y de gobierno. De allí varias investigaciones sobre el proceso de las y opinión pública, se ha dedicado al tema de la opinión pública, como manifestación de la ciudadanía y fortalecimiento de la democracia como Cifuentes (2006), quien presenta y le da importancia al papel que cumple la ciudadanía en el marco de la democracia deliberativa o como lo afirma Niño (1997) las democracias dialógicas o consensuales. Así, que la democracia deliberativa tiene en una de sus manifestaciones en la opinión pública, pero es una opinión pública que no es dirigida y formada intencionalmente por los medios controlados por el poder.

Aquí se podría afirmar que si bien los medios masivos de comunicación controlados por el poder, influyeron en la construcción de una opinión pública tergiversada y manipulada, del proceso de paz llevado a cabo en la Habana (Cuba), desinformando más que informado a los ciudadanos, esta información pudo ser complementada a través de alternativas, un poco más personales, directas y más democráticas; medios lingüísticas, comunicacionales, directos e igualitarios que inciden, también en la formación de opinión pública sobre temas políticos, coyunturales que conciernen al interés de todos y cada uno de los ciudadanos del país, con más razón y frente al tema como era la terminación del conflicto armado con las Farc-ep. Así que "La construcción de los imaginarios sociales y las actitudes frente a la paz y los diálogos necesariamente evolucionan de la mano de la construcción lingüística y comunicativa de la realidad" (Cárdenas, 2013, p. 57). Cabe apuntar a la importancia del proceso de paz en Colombia entre el Gobierno y las Farc-ep.

[Y] por el papel que cumplen los medios de comunicación, en la construcción de sujetos políticos, deben reflexionar sobre la calidad de la información que publican: sobre la información que presentan; su intencionalidad, contenido y estructura con el fin de lograr el objetivo de formar una ciudadanía crítica. Por ello estos medios deben tener presente su función ética y formativa en la tarea que cumplen como educadores políticos y como formadores y estructuradores de la opinión pública (Pino, 2016, p. 95). 
Se hace necesario que todos los actores, incluidos el grupo guerrillero de las Farc-ep, medios de comunicación, quienes los dirigen, la ciudadanía en general y los gobernantes, como facilitadores, educadores formadores en cultura política deben asumir la responsabilidad ética y política de suministrar información objetiva, veraz y confiable sobre los temas de coyuntura e interés para el país, con el fin de cumplir y lograr que la sociedad civil y los sujetos en general sean autónomos, libres y con voz propia, de modo que enriquezcan y participen activa y críticamente en la solución de los problemas de la nación.

\section{Conclusiones}

Estos balances históricos sobre opinión pública y procesos de paz, se coinvierten en unas herramientas para realizar diferentes análisis sobre el proceso de paz, desde su inicio, porque los autores e investigadores consultados presentan en estos estudios diferentes datos, razones, explicaciones y opiniones de los diálogos y sobre todo del logro de la paz, ya que no limitan sus análisis al marco de la relación que se presenta entre la opinión pública y los diálogos de paz, sino que van mucho más allá y advierten que la opinión publica surge y se construye a partir de la información tergiversada o no de los medios de comunicación sobre los diálogos y como esto se convirtió en una estrategia para que los gobiernos legitimaran el procesos.

Así estas investigaciones amplían el campo de análisis político en el país, en tanto muchos estudios muestran que el tema de los diálogos se convirtió y con la ayuda de los medios de comunicación en un dispositivo para legitimar y garantizar la permanencia en el poder de muchos dirigentes. Esta es una de las razones por las cuales los acuerdos de paz firmados recientemente por el gobierno y los FARC-EP y los que están en proceso con el ELN, no pueden analizarse desde una solo perspectiva o desde un solo punto de vista, sino que obliga a los investigadores que se ocupan del tema de la paz a trabajar en coordinación con otras disciplinas, para explicar con mayor rigor científico el fenómeno del logro de la paz en el que está inmerso el país, pues éste, no se explica acudiendo solo, a las relaciones de poder político, sino como una manera de defender ciertos derechos, lograr, reivindicar y reestablecer los ya perdidos y vulnerados.

En este sentido, este estudio proporciona a los lectores un amplio balance de las investigaciones que se han realizado en los últimos años, sobre opinión pública y diálogos de paz: sus perspectivas teóricas, ideológicas y metodológicas, lo que hace de estos trabajos una importante fuente de consulta de un tema sin el cual no es posible comprender, explicar y analizar uno de los fenómenos y acontecimientos sociopolíticos más importantes que por varios años ocupó la atención de todo un país.

Por otra parte, se proporciona un balance de las obras y escritos más importante, que soportan las percepciones, que se manifiestan en la opinión de los colombianos, ubicando al lector y al estudioso, en aquellas obras y productos de investigación de mayor relevancia y actualidad sobre el tema de interés.

Se puede concluir que cada una de estas investigaciones tienen pertinencia y aportan importantes elementos de análisis y argumentos para dar explicación al proceso más pertinente y adecuado para terminar con el fenómeno del conflicto armado, además se resaltar el papel y la función que tiene la opinión pública al momento de legitimar, rechazar, criticar o apoyar un proceso de paz para posteriormente avalar el acuerdo de paz. No obstante, habría que preguntarse por el caso de los medios de comunicación colombiano, especialmente los medios periodísticos, quienes fueron los responsables de cubrir lo que sucedía en la Habana con los diálogos de paz. 
Otro de los asuntos es la falta de un estudio que indague acerca de la opinión de los colombianos sobre la firma del acuerdo de paz actual y más particularmente que se pregunte por la agenda y los puntos aprobados y su impacto en la población desmovilizada, las víctimas y la población colombiana en general. Con la anterior apreciación se podría terminar diciendo que este trabajo y sus hallazgos no agotan el análisis y percepciones que se construyeron los ciudadanos sobre el proceso de paz y la consecución del acuerdo, ni mucho menos definir con exactitud cómo se forma o construye adecuadamente la opinión publica de los colombianos.

$\mathrm{Ni}$ por su puesto agotar el tema sugiriendo que los datos aquí expuestos son definitivos y concluyentes. Es urgente proponer otros puntos de análisis, otras metodologías, acercarse a otros sujetos de estudio y contextos para determinar de una manera más objetiva, como se informan los ciudadanos sobre los problemas y temas más relevantes del país.

\section{REFERENCIAS}

Aguilar, L. (2017). Una reconstrucción del concepto de opinión pública. Revista Mexicana de Opinión Pública, (23), 125-148. Disponible en http://www.revistas.unam.mx/index.php/ rmop/article/view/60908

Aparicio, H. (2004). Medios de comunicación y opinión pública en la sociedad democrática. Revista Venezolana de Ciencias Sociales, 8(2), 322-333. Disponible en http://josemramon.com.ar/wp-content/uploads/Aparicio-AH\%C3\%A9ctor-Medios-de-comunicacion-yopinion-publica-en-la-sociedad.....pdf

Ayala, G. (2015). El proceso de paz de la Habana y las posturas editoriales de la prensa colombiana. Summa Iuris, 3(2), 264-295. Disponible en https://www.funlam.edu.co/revistas/index.php/summaiuris/article/view/1826/1456

Basset, Y. (2018). Claves del rechazo del plebiscito para la paz en Colombia. Estudios Políticos, 52, 241-265. https://doi.org/10.17533/udea.espo.n52a12

Bello, R. (2014). Periodismo y violencia política en Colombia: una mirada crítica desde el periodismo de paz. [Tesis doctoral]. Universidad Austral, Buenos Aires, Argentina. Disponible en https://riu.austral.edu.ar/handle/123456789/235

Cabrales, O. (2012). Ley de justicia y paz y el marco legal para la paz: ¿un paso más hacia la impunidad? Justicia Juris, 8(1), 84-91. https://doi.org/10.15665/rj.v8i1.254

Calderón, J. (2016). Etapas del conflicto armado en Colombia: hacia el posconflicto. Latinoamérica. Revista de Estudios Latinoamericanos, (62), 227-257. Recuperado de http:// latinoamerica.unam.mx/index.php/latino/article/download/49646/49933

Cárdenas, J. (2015). Los medios de comunicación y la construcción de la opinión pública del proceso de paz en la Habana: algunas reflexiones sobre el papel de los medios en la construcción mediática de la realidad en torno a la paz en Colombia. En, ALACIP, VIII Congreso Latinoamericano de Ciencia Política, Pontificia Universidad Católica del Perú, Lima, Perú. Disponible en http://files.pucp.edu.pe/sistema-ponencias/wp-content/ uploads/2014/10/PONENCIA-JUAN-DAVID-CARDENAS-RUIZ-ALACIP-2015.pdf

Cárdenas, J. (2013). Opinión pública y proceso de paz: actitudes e imaginarios de los bogotanos frente al proceso de paz de la habana entre el gobierno colombiano y la guerrilla de las FARC. Ciudad Paz-ando, 6(1), 41-58. https://doi.org/10.14483/udistrital.jour. cpaz.2013.1.a03

Castellano, M., Virviescas, J., Castro, E., Alvarino, C., Pinzón, B. y Gutiérrez, R. (2017). Resolución de conflictos para el fomento de la cultura de paz: importancia de los medios de comunicación alternos en Colombia. Revista Lasallista de Investigación, 14(1), 56-65. https://doi.org/10.22507/rli.v14n1a4 
Charry, C. (2014). Opinión pública y proceso de paz en Colombia en 2014. Anuari del Conflicte Social, (4), 510-545. Disponible en https:/revistes.ub.edu/index.php/ACS/article/ view/12302

Cifuentes, D. (2006). El papel de la ciudadanía en la democracia deliberativa y su relación con la opinión pública. Otras voces, (10), 83-86. https://doi.org/10.7440/res10.2001.08

D'Adamo, O., García, V. y Freidenberg, F. (2000). Medios de comunicación, efectos políticos y opinión pública: Una imagen, vale más que mil palabras? Buenos Aires: Editorial de Belgrano.

Galeano, M. (2004). Estrategias de investigación social cualitativa: El giro en la mirada. Medellín: La carreta.

Galtung, J. (2003). Paz por medios pacíficos: paz y conflicto, desarrollo y civilización. (T. Toda, Trad.) Oslo: Bakeaz-Gernika Gogoratuz.

Glynn, C., Herbst, S., O'Keefe, G., Shapiro, R. \& Lindeman, M. (1999). Public opinión. Boulder: Westview Press

Gómez, M. (2017). Periodismo de paz: formar el camino hacia el final del conflicto. [Tesis de pregrado]. Universidad Colegio Mayor de Nuestra Señora del Rosario, Bogotá, D.C., Colombia. Disponible en http://repository.urosario.edu.co/handle/10336/13656

Gómez, J. (2016). Medios de comunicación en la construcción de paz. HojaLata, (9), 1927. Disponible en http://revistasum.umanizales.edu.co/ojs/index.php/hojalata/article/ view/1786

Gómez, G. (2009). Opinión Pública conocimiento y objeto de conocimiento. Prismasocial, (2), 1-29. Disponible en http://www.isdfundacion.org/publicaciones/revista/pdf/n2_12.pdf

Grajales, G. y Martínez, J. (2020). Tendencias, trayectorias y relaciones de poder en el discurso de construcción de paz en el periódico El Espectador. Ágora USB, 20(1). 112-129. https://doi.org/10.21500/16578031.4184

Jiménez, F. (2009). Hacia un paradigma pacífico: la paz neutra. Convergencia, 16, 141-189.

Libreros, J. (2018). Caracterización de los crímenes de estado y el proceso de paz en Colombia. Pensamiento Americano, 11(22), 119-139. Disponible en https://publicaciones.americana.edu.co/index.php/pensamientoamericano/article/view/265

López, L. y Ramírez, J. (2017). Interferencias en diálogo: representación de la cultura política en mediaciones televisivas, en el marco del Acuerdo de Paz en Colombia. [Tesis de pregrado]. Universidad Pedagógica Nacional, Bogotá, D.C., Colombia. Disponible en http://hdl.handle.net/20.500.12209/9445

Marín, A. y Quintero, J. (2018). Confianza en el proceso de paz en Colombia en twitter. Revista Mexicana de Sociología, 80(1), 115-137. Disponible en http://revistamexicanadesociologia.unam.mx/index.php/rms/article/view/57705

Montoya, A. (Ed.) (2005). Percepciones y opiniones de los colombianos sobre justicia, verdad, reparación y reconciliación. Bogotá, D.C.: Fundación social y la Consejería en Proyectos. Recuperado de https://www.ictj.org/sites/default/files/ICTJ-Colombia-PerceptionsOpinions-2005-Spanish.pdf

Morales, J., Rodríguez, E., Reyes, M. y O’Quinn, J. (2011). Opinión pública y democracia, algunas aportaciones para su estudio. Espacios Públicos, 14(32), 183-205. Disponible en http://hdl.handle.net/20.500.11799/39729

Niño, C. (1997). La constitución de la democracia deliberativa. Barcelona: Gedisa.

Palacio, J. (2017). Perspectivas y comparaciones en los diálogos de paz en Colombia. Revista Folhmyp, (9), 95-100. Disponible en https://revistas.pedagogica.edu.co/index.php/FHP/ article/view/8551 
Pedroza, L. (2011). Opinión pública en Colombia: Análisis de los medios de comunicación: Semana y Cambio en el cubrimiento periodístico del tema de justicia y paz durante el año 2005-2006”. [Tesis de grado]. Universidad de Antioquia, Medellín, Colombia.

Pino, M. (2016). Construcción de opinión pública en los estudiantes de ciencia política, de la universidad de Antioquia, sobre los puntos de la agenda: desarrollo agrario y reparación a las víctimas, discutidos en el actual proceso de paz entre el gobierno y las Farc-ep. [Tesis de pregrado]. Universidad de Antioquía, Medellín, Colombia. Disponible en http:// bibliotecadigital.udea.edu.co/bitstream/10495/12613/1/PinoMontoya_2016_ConstruccionOpinionPublica.pdf

Ramírez, V., Ceballos, J. y Molina, N. (2019). Actitudes de la población vallecaucana frente a la convivencia y reconciliación en el proceso de paz entre el Gobierno y las Fuerzas Armadas Revolucionarias de Colombia (FARC-EP). Katharsis, (28), 3-9.

Ramos, E. (2016). El proceso de construcción de paz colombiano más allá de la negociación: una propuesta desde la paz transformadora y participativa. Ágora USB, 16(2), 513-532. https://doi.org/10.21500/16578031.2458

Ramos, E. (2015). Paz transformadora (y participativa): teoría y método de la paz y el conflicto desde la perspectiva sociopráxica. Tegucigalpa: Instituto Universitario de Democracia, Paz y Seguridad (IUDPAS).

República de Colombia. Farc-Ep. (2016). Acuerdo final para la terminación del conflicto y la construcción de una paz estable y duradera. [Online]. Recuperado de https://www.jep.gov. co/Marco\%20Normativo/Normativa_v2/01\%20ACUERDOS/N01.pdf

Sartori, G. (1998). Homo videns: La sociedad teledirigida. Buenos Aires: Taurus.

Semana. (septiembre 1, 2012). Los puntos de la agenda. Semana. [Online]. Disponible en http://www.semana.com/nacion/articulo/los-puntos-agenda/263987-3

Ugarriza, J. Cotrina, A. y Sequera, N. (2013). ¿Qué se negocia en los procesos de paz? : Agendas y factores de éxito 1989-2012. Análisis político, (77), 153-174. Disponible en https:// revistas.unal.edu.co/index.php/anpol/article/view/44119/45368

Valencia, A. (2008). Historia de Guerra y paz en el caguan, análisis comparativo. Miami: Interamerican Democratic institute.

Villa, J. y Arroyave, L. (2018). Creencias sociales y orientaciones emocionales colectivas sobre la paz negociada en ciudadanos de Medellín. Kavilando, 10(21), 449-469. Disponible en http://kavilando.org/revista/index.php/kavilando/article/view/272

Villarraga, A. (2015). Los procesos de paz en Colombia 1982-2014. [Documento resumen]. Bogotá, D.C.: Fundación Cultura Democrática.

Jose Wilmar Pino Montoya recibió el grado de Doctor en Filosofía el 21 de octubre de 2011 de la Universidad Pontificia Bolivariana (Medellín, Colombia). Sus intereses de investigación han incluido la filosofía política, la educación, los temas de familia, la metodología de la investigación y la filosofía. Investigador de la Facultad de Educación y Humanidades de la Universidad Católica Luis Amigó (Colombia). https://orcid.org/0000-0001-9035-2058

Melina Castaño Gómez es Politóloga de la Universidad de Antioquia (Colombia) con Diplomado Equidad del Género. Diplomado en Planeacion de proyectos y gestion publica. Diplomado en metodología, gestion y diseño de encuestas. Auxiliar de investigación (Colombia). https://orcid.org/0000-0002-2354-4014 